\title{
Application of bone turnover markers and DXA and QCT in an elderly Chinese male population
}

\author{
Yuan Yuan", Ping Zhang", Wei Tian, Xiaohui Deng, Rui Yue, Xiaozhu Ge, Xiaoyu Li \\ Department of Geriatrics, Beijing Jishuitan Hospital, Beijing, China \\ Contributions: (I) Conception and design: Y Yuan, P Zhang; (II) Administrative support: X Li; (III) Provision of study materials or patients: W Tian; \\ (IV) Collection and assembly of data: X Deng, R Yue; (V) Data analysis and interpretation: X Ge; (VI) Manuscript writing: All authors; (VII) Final \\ approval of manuscript: All authors. \\ \#These authors contributed equally to this work and are co-first authors. \\ Correspondence to: Xiaoyu Li. Department of Geriatrics, Beijing Jishuitan Hospital, Beijing 100035, China. Email: yuan100yuan100@163.com.
}

Backgroundk To investigate the clinical values and relationships of bone turnover markers (BTMs), dualenergy X-ray absorptiometry (DXA), and quantitative computed tomodensitometry (QCT) in the screening of osteoporosis (OP) in elderly Chinese males.

Methods: General data, including age, height, and weight, the results of BTM measurements, and the findings of DXA and QCT in 357 male patients aged $\geq 50$ years who visited the outpatient or inpatient Department of Geriatrics, Beijing Jishuitan Hospital from June 2017 to May 2019 were retrospectively analyzed.

Results: The OP detection rates based on T-scores of DXA $\mathrm{L}_{1-4}$, DXA total hip, and spine QCT were 3.4\% (12/357), 13.2\% (47/357), and 40.3\% (144/357), respectively. QCT had a significantly higher OP detection rate than did DXA $(\mathrm{P}<0.001)$. There were 24 cases of fragility fractures, which were significantly correlated with the DXA total hip BMD and its T-score, with risk cut-off values of $0.607 \mathrm{~g} / \mathrm{cm}^{2}$ and -2.950 , respectively. The measured levels of the 5 BTMs were as follows: total procollagen type I amino-terminal propeptide (tPINP), $39.23 \pm 20.82 \mathrm{ng} / \mathrm{mL}$; $\beta$-isomerized C-terminal telopeptides ( $\beta$-CTX), $0.38 \pm 0.21 \mathrm{ng} / \mathrm{mL}$; osteocalcin $(\mathrm{OC}), 13.50 \pm 8.80 \mathrm{ng} / \mathrm{mL} ; 25$-hydroxycholecalciferol $\left(25(\mathrm{OH}) \mathrm{D}_{3}\right), 12.90 \pm 7.46 \mathrm{ng} / \mathrm{mL}$; and parathormone $(\mathrm{PTH}), 54.50 \pm 25.35 \mathrm{pg} / \mathrm{mL}$. The elevation of tPINP, $\beta$-CTX, and OC were negatively correlated with aging and positively correlated with decreased BMD (all $\mathrm{P}<0.05$ ). $\mathrm{OC}$ and $25(\mathrm{OH}) \mathrm{D}_{3}$ values were significantly lower than their normal range. Among the 43 patients with normal bone mass on both DXA and QCT examinations, 34 presented with abnormal BTMs, including elevated tPINP in 2 cases, elevated $\beta$-CTX in 2 cases, and OC decreased in 31 cases.

Conclusions: In the Chinese elderly male population, spine QCT has a higher detection rate of OP than DXA, whereas hip DXA is more advantageous in predicting the risk of fragility fracture. tPINP, $\beta$-CTX, and OC can be used as reliable indicators for the dynamic observation of bone content changes and may screen for early bone metabolism abnormalities when BMD examinations still show negative results.

Keywords: Elderly males; dual-energy X-ray absorptiometry (DXA); quantitative computed tomodensitometry (QCT); bone mineral density (BMD); bone turnover markers (BTMs)

Submitted Jan 20, 2021. Accepted for publication Jun 03, 2021.

doi: 10.21037/apm-21-612

View this article at: https://dx.doi.org/10.21037/apm-21-612 


\section{Introduction}

Arising from a variety of factors, osteoporosis (OP) consists of a group systemic bone diseases and is characterized by reduced bone strength and increased likelihood of fractures. Due to the lack of specific clinical symptoms in the early stages of OP, fragility fracture is typically the most common cause of presentation to hospitals (1). Fragility fracture not only significantly increases the short-term (within 3 months after fracture) all-cause mortality rate (2) but also dramatically increases the long-term disability rate of patients, bringing heavy burden on patients' families and society at large.

China has the largest elderly population and the highest number of OP patients worldwide. Postmenopausal women are 5-10 times more likely to develop OP than men, and therefore most previous studies on OP have been performed in female populations. In recent years it has been found that $\mathrm{OP}$ in men is not uncommon, with its incidence showing a significant upward trend (3-6). Studies have even found that the incidence of postoperative complications and mortality rates in males with hip fracture are significantly higher than those in women, suggesting that male osteoporotic patients have a poor prognosis $(7,8)$.

Therefore, early diagnosis, timely treatment, and effective prevention of fragility fractures have become major challenges for clinicians and researchers. Current techniques used to diagnose OP include dual-energy X-ray absorptiometry (DXA) (9), quantitative computed tomodensitometry (QCT) (10), and ultrasound (US) (11). DXA remains the gold standard for the diagnosis of OP; however, although DXA is widely available, its detection performance is relatively limited. The previous study of our hospital found that QCT had a higher OP detection rate than did DXA (12).

Bone turnover marker (BTM) measurement has been widely adopted in clinical settings in recent years and plays an important role in determining treatment regimens or assessing short-term treatment efficacies (13). However, few studies have explored the roles of BTMs in the diagnosis of OP.

Here we applied the findings of DXA and QCT, as well as the results of BTM measurement, to investigate the bone mass changes and related bone metabolic parameters in an elderly male population in Beijing, with an attempt to inform early OP screening and prevention.

We present the following article in accordance with the MDAR reporting checklist (available at https://dx.doi. org/10.21037/apm-21-612).

\section{Methods}

\section{Subjects}

The study was approved by the ethics committee of Beijing Jishuitan Hospital (No. 201907-09) and complied with ethical principles of the Declaration of Helsinki (as revised in 2013). Individual consent for this retrospective analysis was waived.

The inclusion criteria were as follows: (I) males; (II) aged $\geq 50$ years; (III) residing in Beijing for a long period of time; and (IV) outpatients or inpatients in the Department of Geriatrics of Beijing Jishuitan Hospital between June 2017 and May 2019.

The exclusion criteria included the following: (I) with metabolic/endocrine disorders, blood diseases, liver and kidney diseases, and/or connective tissue diseases; (II) having undergone gastrectomy; and/or (III) use of glucocorticoids, sex hormones, thyroid hormones, and/or other drugs affecting bone metabolism.

\section{Survey items}

\section{General data}

A total of 357 patients meeting the criteria were included. These patients were aged $74.68 \pm 16.16$ years, with a body mass index (BMI) of 23.91 \pm 3.27 . DXA, QCT, and BTM measurements were completed during the same outpatient visit or hospital stay.

\section{DXA and interpretation of its results}

Lumbar spine $\left(\mathrm{L}_{1-4}\right)$ and right hip bone mineral density (BMD) were regularly measured by DXA using GE Lunar Prodigy DXA scanners (GE Lunar Prodigy and DPX Bravo DXA scanners, GE Healthcare, WI, USA). Vertebrae with significant fractures or deformities were excluded manually by the researchers. The right hip measured included the femoral neck, the greater trochanter, and the Ward triangle.

Interpretation of results was done according to the World Health Organization (WHO) osteoporosis diagnostic classification assessment [1994] (14), in which OP is defined as a $\mathrm{T}$ score of 2.5 or more standard deviations (SDs) below the mean (i.e., $\leq-2.5$ ), osteopenia as a $\mathrm{T}$ score of -1.0 to $-2.5 \mathrm{SD}$, and normal bone mass as a $\mathrm{T}$ score of -1.0 or higher SD.

\section{QCT and interpretation of its results}

QCT was performed using a Toshiba Aquilion 64-row spiral CT scanner (Toshiba, Tokyo, Japan) with a Mindways 
5-sample solid-state calibration phantom (Mindways, Austin, TX, USA). All analyses of raw data were performed using the Mindways QCT Pro software calibrated with a QCT phantom (Mindways Software, Inc., Austin, TX, USA). BMD from $L_{1}$ to $L_{4}$ and their adjacent vertebral bodies were measured separately. The areas of interest were marked in the middle of the vertebral body $\left(\mathrm{L}_{2-4}\right)$, avoiding the cortical bones and the basivertebral plexus.

Interpretation of results was done according to the International Society for Clinical Densitometry (ISCD), and the American College of Radiology cutoff values for lumbar spine QCT $(15,16)$ were used to categorize patients as $\mathrm{OP}\left(<80 \mathrm{mg} / \mathrm{cm}^{3}\right)$, osteopenia $\left(80-120 \mathrm{mg} / \mathrm{cm}^{3}\right)$, or normal bone mass $\left(>120 \mathrm{mg} / \mathrm{cm}^{3}\right)$.

\section{BTMs}

Fasting blood samples were collected in the early morning (from 6 am to 9 am) and analyzed using a Roche COMBAS e601 autoanalyzer (Roche Diagnostics, Basel, Switzerland) with the standard Cobas kit (Roche, Shanghai, China). The precision was validated by two consecutive measurements of the same sample, with the correction factor being \pm 3 SD.

The BTMs and their normal ranges were as follows: (I) total procollagen type I amino-terminal propeptide (tPINP) $(15.3-52.7 \mathrm{ng} / \mathrm{mL})$; (II) $\beta$-isomerized C-terminal telopeptides ( $\beta$-CTX) $(<0.854 \mathrm{ng} / \mathrm{mL}$ in males); (III) osteocalcin (OC) (15-46 ng/mL); (IV) 25 -hydroxycholecalciferol $\left(25(\mathrm{OH}) \mathrm{D}_{3}\right)(20-40 \mathrm{ng} / \mathrm{mL})$; and (V) parathormone $(\mathrm{PTH})(15-65 \mathrm{pg} / \mathrm{mL})$.

\section{Statistical analysis}

Statistical analysis was performed using SPSS 19.0 19.0 software (IBM Corp., Armonk, NY, USA). All the measurement data were tested for normality. For normally distributed samples, ANOVA was used for multiple comparisons; non-normally distributed data were analyzed by rank-sum test. Count data were compared using the chi-square test. The significance level was set at a $\mathrm{P}$ value $<0.05$.

\section{Results}

\section{Results of BMD measurements}

Spinal DXA ( $\mathrm{L}_{1-4} \mathrm{~T}$-scores) showed normal bone mass in 304 cases (85.1\%), osteopenia in 41 cases (11.5\%), and OP in 12 cases (3.4\%). Hip DXA (total hip T-score) showed normal bone mass in 177 cases $(49.6 \%)$, osteopenia in 133 cases $(37.2 \%)$, and OP in 47 cases $(13.2 \%)$. Spinal QCT $\left(\mathrm{L}_{2-4} \mathrm{BMD}\right)$ showed normal bone mass in 83 cases $(23.3 \%)$, osteopenia in 130 cases $(36.4 \%)$, and OP in 144 cases $(40.3 \%)$. There were statistically significant differences (all $\mathrm{P}<0.05$ ) in the detection rate of bone mass abnormalities among the above three criteria (Table 1). The bone mass abnormalities detected by DXA and QCT are shown in Table 1.

\section{Correlation between bone density and fragility fractures}

Fragility fractures occurred in 24 of 357 cases, including 7 cases of hip fractures, 7 cases of thoracolumbar fractures, 4 cases of rib fractures, 3 cases of patella fractures, 2 cases of ankle fractures, and 1 case of wrist fracture. For these 24 patients with fragility fractures, the DXA $\mathrm{L}_{1-4} \mathrm{~T}$-score criteria indicated 2 cases (8.3\%) had OP, 6 cases $(25.0 \%)$ had osteopenia, and 16 cases $(66.7 \%)$ had normal bone mass; the DXA total hip T-score criteria showed 10 cases (41.7\%) had OP, 14 cases (58.3\%) had osteopenia, and 0 case had normal bone mass; the QCT BMD values showed 13 cases (54.5\%) had OP, 11 cases (45.5\%) had osteopenia, and 0 cases had normal bone mass.

Further comparison of the bone density between the fragility fracture group and the nonfracture group yielded statistically significant differences in the DXA total hip BMD values and T-scores (Table 2).

The receiver operating characteristic (ROC) curves showed a cutoff value of $0.607 \mathrm{~g} / \mathrm{cm}^{2}$ (sensitivity 0.917 and specificity 0.995 ) for the DXA total hip BMD and a cutoff value of -2.950 (sensitivity 0.917 and specificity 0.995 ) for the T-score.

\section{Correlation analysis of BTMs}

The mean values of BTMs in 357 patients were as follows: tPINP, $39.23 \pm 20.82 \mathrm{ng} / \mathrm{mL}$; $\beta$-CTX, $0.38 \pm 0.21 \mathrm{ng} / \mathrm{mL}$; OC, $13.50 \pm 8.80 \mathrm{ng} / \mathrm{mL} ; 25(\mathrm{OH}) \mathrm{D}_{3}, 12.90 \pm 7.46 \mathrm{ng} / \mathrm{mL}$; and PTH, $54.50 \pm 25.35 \mathrm{pg} / \mathrm{mL}$. Among them, tPINP, $\beta$-CTX, and PTH were basically in the normal ranges, while $\mathrm{OC}$ and $25(\mathrm{OH}) \mathrm{D}_{3}$ were significantly lower than the lower limit of normal (LLN). tPINP, $\beta$-CTX, and OC were significantly correlated with BMD. Among the BMD criteria, DXA total hip T-score had the most significant correlation with these 3 BTMs $(r=-0.235, \mathrm{P}<0.01 ; \mathrm{r}=-0.304$, $\mathrm{P}<0.01 ; \mathrm{r}=-0.257, \mathrm{P}<0.01$, respectively). $25(\mathrm{OH}) \mathrm{D}_{3}$ and 
Table 1 Comparisons of the results of 3 different bone mineral density examinations in 357 patients

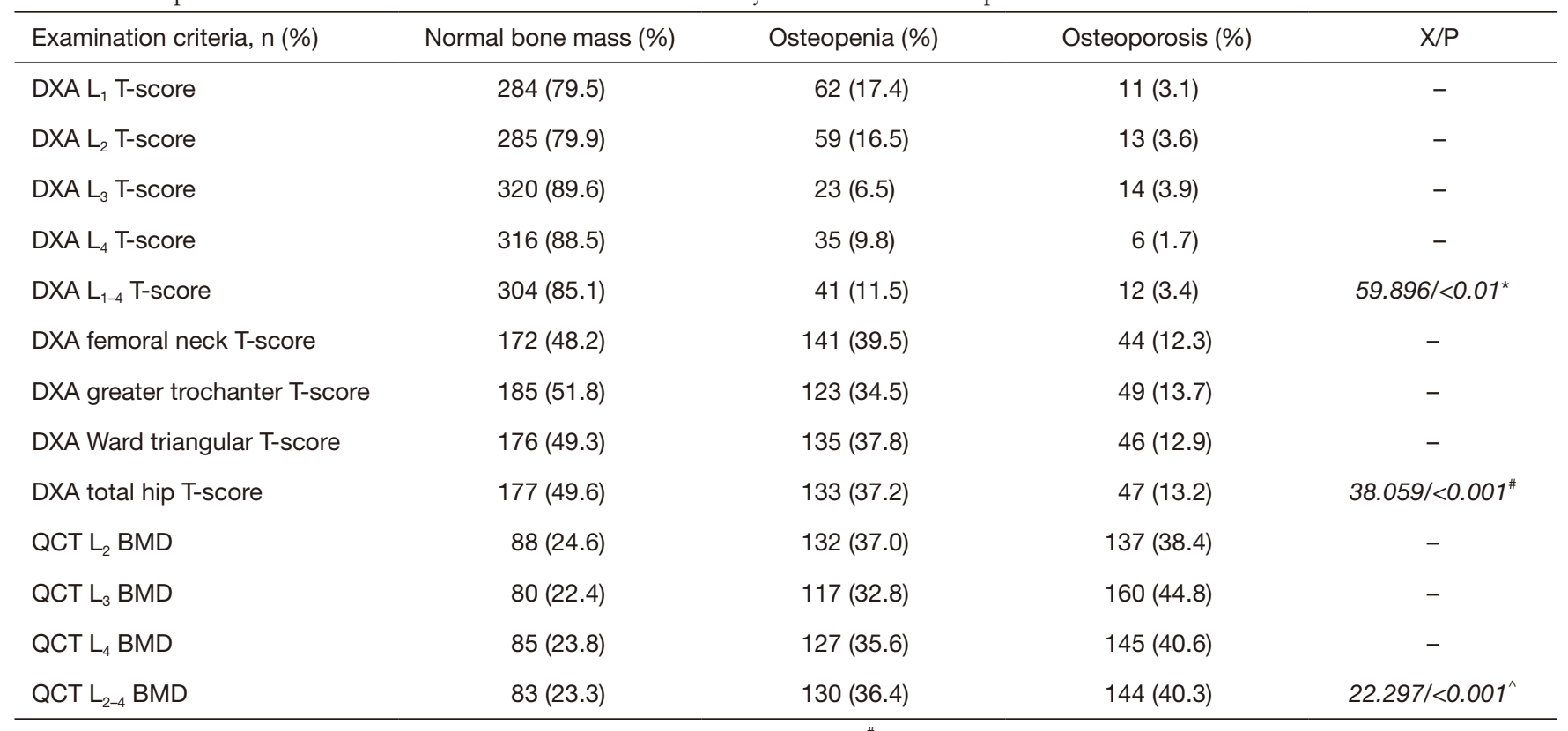

*, intergroup comparison of DXA $\mathrm{L}_{1-4}$ T-scores with DXA total hip T-scores; *, intergroup comparison of DXA total hip T-scores with QCT

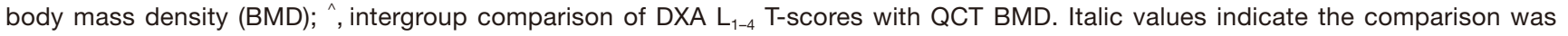
statistically significant. DXA, dual-energy X-ray absorptiometry; QCT, quantitative computed tomodensitometry.

Table 2 Comparison of BMD between fragility fracture group and non-fracture group

\begin{tabular}{lcccc}
\hline Examination $($ mean $\pm \mathrm{SD})$ & Unit & Non-fracture group & Fragility fracture group & F/P \\
\hline DXA L $_{1-4}$ & $\mathrm{~g} / \mathrm{cm}^{2}$ & $1.206 \pm 0.230$ & $1.164 \pm 0.200$ & $0.386 / 0.535$ \\
& T-score & $1.033 \pm 1.810$ & $0.683 \pm 1.639$ & $0.426 / 0.514$ \\
DXA total hip & $\mathrm{g} / \mathrm{cm}^{2}$ & $0.939 \pm 0.151$ & $0.813 \pm 0.132$ & $7.989 / 0.005$ \\
& T-score & $-0.386 \pm 1.170$ & $-1.367 \pm 1.031$ & $8.033 / 0.005$ \\
QCT L L-4 & $\mathrm{mg} / \mathrm{cm}^{3}$ & $94.467 \pm 35.279$ & $83.955 \pm 29.390$ & $0.935 / 0.335$ \\
& T-score & $-2.900 \pm 1.258$ & $-3.358 \pm 1.057$ & $1.375 / 0.242$ \\
\hline
\end{tabular}

Italic values indicate the comparison was statistically significant. BMD, bone mineral density; DXA, dual-energy X-ray absorptiometry; QCT, quantitative computed tomodensitometry.

PTH levels showed no definite correlation with BMD values (Table 3).

Both QCT BMD values and DXA hip total T-scores suggested normal bone mass in 43 cases, among whom both tPINP and $\beta$-CTX were higher than the upper limit of normal (ULN) in 2 cases (tPINP: 86.87 and $61.78 \mathrm{ng} / \mathrm{mL}$; $\beta$-CTX: 0.97 and $0.86 \mathrm{ng} / \mathrm{mL})$. No patient had a tPINP below LLN. OC was below LLN in 31 cases but did not exceed ULN in any patient. Thus, tPINP, $\beta$-CTX, and OC might be advantageous for the early detection of abnormal bone density.

\section{Correlations of age and BMI with BMD and BTMs}

Age was positively correlated with $\mathrm{DXA} \mathrm{L}_{1-4} \mathrm{BMD}$ values and their T-scores $(\mathrm{r}=0.188, \mathrm{P}=0.002 ; \mathrm{r}=0.169, \mathrm{P}=0.005)$ and negatively correlated with DXA total hip BMD values and their T-scores $(r=-0.122, \mathrm{P}=0.45 ; \mathrm{r}=-0.173, \mathrm{P}=0.004)$ and QCT BMD values and their T-scores $(\mathrm{r}=-0.161, \mathrm{P}=0.016$; $\mathrm{r}=-0.177, \mathrm{P}=0.008)$.

Among the BTMs, tPINP, $\beta$-CTX, and OC showed significant negative correlations with age $(r=-0.213$, $\mathrm{P}<0.01 ; \mathrm{r}=-0.219, \mathrm{P}<0.01 ; \mathrm{r}=-0.151, \mathrm{P}=0.01)$; however, 
Table 3 Analysis of bone metabolism levels based on bone mineral density grades

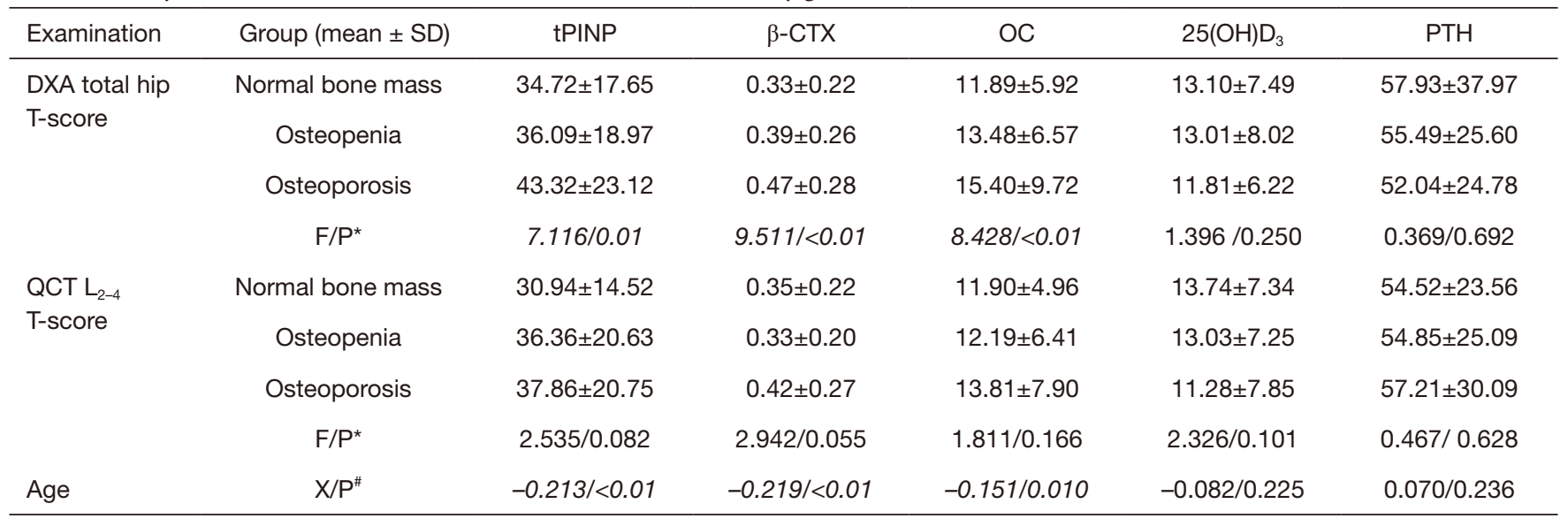

*, ANOVA; ", Chi-square test. Italic values indicate the comparison was statistically significant. DXA, dual-energy X-ray absorptiometry; QCT, quantitative computed tomodensitometry; tPINP, total procollagen type I amino-terminal propeptide; OC, osteocalcin; PTH, parathormone.
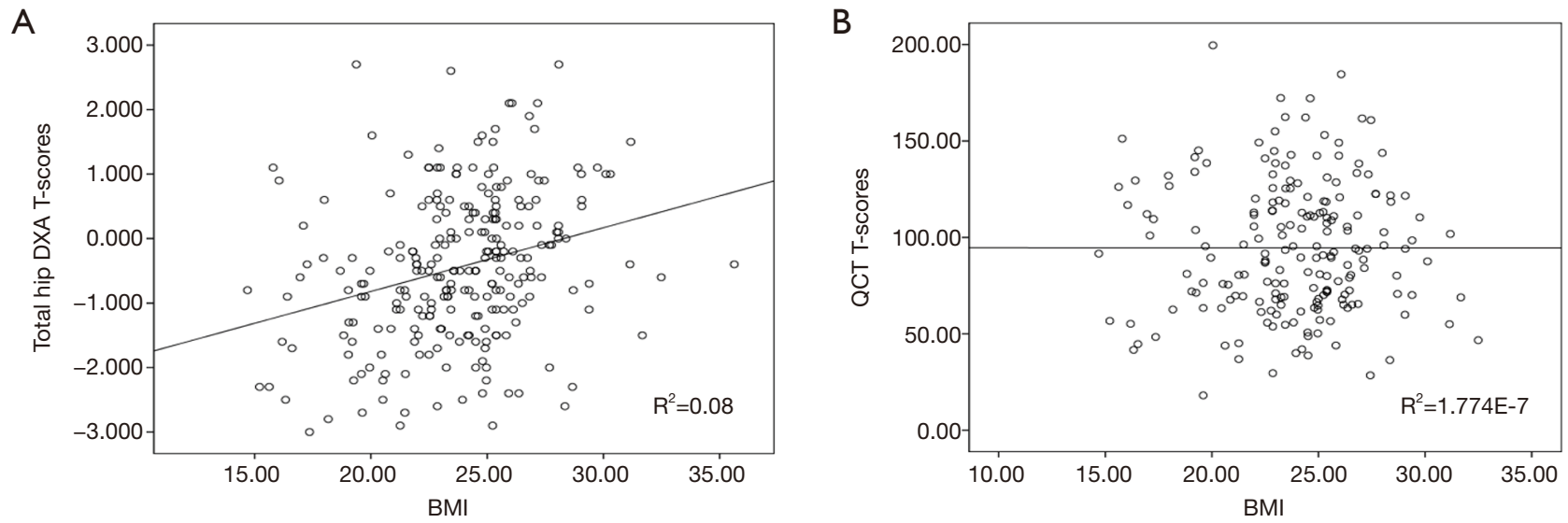

Figure 1 Scatterplot of the relationships of BMI with DXA and QCT T-score. (A) Relationship between BMI and DXA total hip T-score; (B) relationship between BMI and QCT total T-score. BMI, body mass index; DXA, dual-energy X-ray absorptiometry; QCT, quantitative computed tomodensitometry.

PTH and $25(\mathrm{OH}) \mathrm{D}_{3}$ were not significantly correlated with age (Table 3).

BMI was positively correlated with DXA BMD measurements (DXA $\mathrm{L}_{1-4}$ and DXA total hip $\mathrm{BMD}$ values and T-scores; both $\mathrm{P}$ values <0.01); however, it showed no significant correlation with QCT findings (Figure 1).

\section{Discussion}

It is estimated that worldwide, 1 in 5 men over the age of 50 will experience OP fractures in their remaining lifetime (17), and the fractures in males mainly occur within the last 10 years of life (18). The excessively high prevalence and mortality rates of osteoporotic fractures in men have led to tremendous individual and socioeconomic burden.

In our current study, the incidence of OP in men over 50 years of age was $3.4 \%$ (spinal DXA) and $13.2 \%$ (hip DXA), which is similar to previous reports in China (range, $2.0-13.4 \%)(6,12,19,20)$; QCT increased the detection rate to $40.3 \%$. The incidence of fragility fractures was $6.7 \%$ (24/357 cases), and more than half of them were hip fractures and thoracolumbar fractures $(58.3 \%, \mathrm{n}=14)$. The hip DXA was significantly correlated with the occurrence 
of fragility fracture, and the hip DXA cutoff value (BMD, $0.607 \mathrm{~g} / \mathrm{cm}^{2}$; T-score, -2.950 ) may be used as a risk predictor of fragility fracture in clinical practice.

\section{DXA versus QCT: which one is better?}

Both DXA and QCT have their advantages and disadvantages. (I) DXA uses a low dose of radiation and is relatively easy to operate. However, it can only measure bone density in 2 dimensions $\left(\mathrm{mg} / \mathrm{cm}^{2}\right)$ and thus can be easily influenced by factors such as bone size, spinal degeneration, vertebral compression and deformation, and abdominal aortic calcification, which often result in misjudgment, especially for the elderly. (II) QCT is a 3-dimensional measurement of bone density $\left(\mathrm{mg} / \mathrm{cm}^{3}\right)$ and thus will not be influenced by adjacent tissues. In our current study, QCT had a higher detection rate of OP in elderly patients than spinal and hip DXA. However, it has several drawbacks, including relatively high radiation exposure and high expenditure.

Although QCT is superior to DXA in terms of population detection rate, 36 patients in our current analysis had normal spinal QCT findings but had abnormal bone mass on hip DXA (including 3 cases of OP and 33 cases of osteopenia). The possible explanation may be as follows: the morphology of the proximal femur is less variable and the repeatability of the examinations is good; furthermore, there are fewer osteophytes and more significant bone loss in the proximal femur. Therefore, both lumbar spine QCT and hip DXA are recommended bone density examinations in the diagnosis of $\mathrm{OP}$ in elderly men. Actually, they can act in a complementary manner.

\section{BTMs}

\section{5(OH)D3 and PTH}

Both $25(\mathrm{OH}) \mathrm{D}_{3}$ and PTH are calcium- and phosphorusregulating hormones. Although vitamin $\mathrm{D}_{3}$ level was not found to be significantly associated with aging and decreased BMD in elderly men, severe vitamin $\mathrm{D}$ deficiency is prevalent and can exacerbate the occurrence and development of OP in the elderly. Vitamin D deficiency is associated with multiple factors such as decreased outdoor activities, poor intake of vitamin $\mathrm{D}$, decreased renal function, and insufficient synthesis in the body.

PTH is a peptide hormone secreted from the parathyroid glands and can stimulate bone resorption. There are three forms of PTH fragments in the blood circulation, namely intact PTH, N-terminal PTH fragments, and C-terminal PTH fragments. The intact PTH can be detected in our center. Immunoreactive PTH increases gradually with age in healthy individuals in adulthood; decreased renal function and increased blood phosphorus in the elderly can also be secondary to a rise in PTH, and the overproduction of PTH can lead to increased bone conversion and enhanced bone breakdown and resorption, resulting in the development of age-related OP (21). The present study showed that PTH levels in elderly men were close to the upper limit of normal values, yet no clear correlation between PTH and aging was found, which might be explained by the interference by multiple factors such as vitamin D deficiency and low blood calcium and by the relatively small sample size. The reference range of PTH has been lowered in international studies (22). Currently, the acceptable reference range for PTH is between 10 and $60 \mathrm{pg} / \mathrm{mL}$, which is lower than our reference values. It has been proposed that a PTH of $>50 \mathrm{pg} / \mathrm{mL}$ in the presence of normal renal function and elevated serum calcium strongly suggests primary hyperparathyroidism (23). Thus, the potential disease risk should be assessed in a more comprehensive manner even if the PTH level is within the normal range.

\section{BTMs reflecting bone formation}

More than $90 \%$ of the osteoid matrix of bone consists of type I collagen. Type I procollagen contains $\mathrm{N}$ - and C-terminal extensions, which are removed by specific proteases during the conversion of procollagen to collagen. The tPINP level reflects the synthesis of collagen type I, a key step in bone transformation. Elevated tPINP level indicates increased synthesis of type I collagen and active bone turnover. Measurement of serum tPINP levels is of great clinical value for the identification and typing of senile OP. In our current study, elevated tPINP was closely correlated with decreased BMD, which, together with its advantage of rapid response to changes in skeletal physiology, may be important for its future use in the management of elderly male osteoporotic patients.

$\mathrm{OC}$ is one of the most important noncollagenous proteins in the bone matrix. It is currently believed that the concentration of $\mathrm{OC}$ in blood reflects the rate of bone formation. When bone formation is coupled with bone resorption, $\mathrm{OC}$ is an indicator of bone conversion; in contrast, when bone formation is uncoupled from bone resorption, OC is an indicator of bone formation. Measurement of serum osteocalcin levels is clinically valuable for the identification and typing of senile $\mathrm{OP}$ 
and for the evaluation of therapeutic efficacy (24). In our current study, OC levels were significantly lower than the normal range in the elderly male population and correlated significantly with aging and decreased $\mathrm{BMD}$, indicating that bone conversion decreases in men with aging, in which OP patients showed relatively active but dysregulated bone formation and bone resorption.

\section{BTMs reflecting bone resorption}

$\beta$-CTX is a degradation product of type I collagen during bone metabolism. Notably, C-telopeptide [C-terminal telopeptide of type 1 collagen $(\mathrm{CTx})$ ] is a specific marker for bone resorption. Measurement of serum $\beta$-CTX levels is clinically valuable for assessing bone absorption in patients with senile $\mathrm{OP}$ and for the monitoring of therapeutic efficacy. In our current study, $\beta$-CTX was significantly correlated with decreased BMD.

Among 43 elderly men with normal bone mass, 34 showed abnormally elevated tPINP, abnormally elevated $\beta$-CTX, or abnormally decreased OC. Measurement of these 3 BTMs may be an important means of identifying bone loss in the very early stages when imaging does not yet show a decrease in bone mass.

In conclusion, spinal QCT has a higher detection rate of OP than does DXA, whereas hip DXA is more advantageous in predicting the risk of fragility fracture. tPINP, $\beta$-CTX, and OC can be used as reliable indicators for the dynamic observation of bone content changes and may screen for early bone metabolism abnormalities when BMD examinations still show negative results.

\section{Acknowledgments}

Funding: This work was supported by grants from the Beijing Municipal Administration of Hospitals Incubating Program (No. PZ2021004) and the Research Fund of the Chinese Health Management Doctoral Alliance (2020).

\section{Footnote}

Reporting Checklist: The authors have completed the MDAR reporting checklist. Available at https://dx.doi. org/10.21037/apm-21-612

Data Sharing Statement: Available at https://dx.doi. org/10.21037/apm-21-612

Conflicts of Interest: All authors have completed the ICMJE uniform disclosure form (available at https://dx.doi. org/10.21037/apm-21-612). All authors report funding from Beijing Municipal Administration of Hospitals Incubating Program (No. PZ2021004) and the Research Fund of the Chinese Health Management Doctoral Alliance (2020). The authors have no other conflicts of interest to declare.

Ethical Statement: The authors are accountable for all aspects of the work in ensuring that questions related to the accuracy or integrity of any part of the work are appropriately investigated and resolved. The study was approved by the ethics committee of Beijing Jishuitan Hospital (No. 201907-09) and complied with ethical principles of the Declaration of Helsinki (as revised in 2013). Individual consent for this retrospective analysis was waived.

Open Access Statement: This is an Open Access article distributed in accordance with the Creative Commons Attribution-NonCommercial-NoDerivs 4.0 International License (CC BY-NC-ND 4.0), which permits the noncommercial replication and distribution of the article with the strict proviso that no changes or edits are made and the original work is properly cited (including links to both the formal publication through the relevant DOI and the license). See: https://creativecommons.org/licenses/by-nc-nd/4.0/.

\section{References}

1. Rachner TD, Khosla S, Hofbauer LC. Osteoporosis: now and the future. Lancet 2011;377:1276-87.

2. Haentjens P, Magaziner J, Colón-Emeric CS, et al. Meta-analysis: Excess Mortality After Hip Fracture Among Older Women and Men. Ann Intern Med 2010;152:380-90.

3. Wang Y, Tao Y, Hyman ME, et al. Osteoporosis in China. Osteoporos Int 2009;20:1651-62.

4. Zhu H, Fang J, Luo X, et al. A survey of bone mineral density of healthy Han adults in China. Osteoporos Int 2010;21:765-72.

5. Chen P, Li ZZ, Hu Y. Prevalence of osteoporosis in China: a meta-analysis and systematic review. BMC Public Health 2016;16:1039.

6. Cui Z, Meng X, Feng H, et al. Estimation and projection about the standardized prevalence of osteoporosis in mainland China. Arch Osteoporos 2019;15:2 .

7. Hu F, Jiang C, Shen J, et al. Preoperative predictors for mortality following hip fracture surgery: a systematic 
review and meta-analysis. Injury 2012;43:676-85.

8. Flikweert ER, Wendt KW, Diercks RL, et al. Complications after hip fracture surgery: are they preventable?. Eur J Trauma Emerg Surg 2018;44:573-80.

9. Wahner HW. Clinically useful and readily available techniques for measurements of bone mineral and body composition by photon or X-ray absorptiometry. Trends Endocrinol Metab 1990;1:382-87.

10. Smith DA, Hosie CJ, Deacon AD, et al. Quantitative gamma-ray computed tomography of the radius in normal subjects and osteoporotic patients. Br J Radiol 1990;63:776-82.

11. McCloskey EV, Murray SA, Charlesworth D, et al. Assessment of broadband ultrasound attenuation in the os calcis in vitro. Clin Sci (Lond) 1990;78:221-5.

12. Xu XM, Li N, Li K, et al. Discordance in diagnosis of osteoporosis by quantitative computed tomography and dual-energy X-ray absorptiometry in Chinese elderly men. J Orthop Translat 2018;18:59-64.

13. Nishizawa Y, Miura M, Ichimura S, et al. Executive summary of the Japan Osteoporosis Society Guide for the Use of Bone Turnover Markers in the Diagnosis and Treatment of Osteoporosis (2018 Edition). Clin Chim Acta 2019;498:101-7.

14. Kanis JA, Melton LJ 3rd, Christiansen C, et al. The diagnosis of osteoporosis. J Bone Miner Res 1994;9:1137-41.

15. Engelke K, Adams JE, Armbrecht G, et al. Clinical use of quantitative computed tomography and peripheral quantitative computed tomography in the management of osteoporosis in adults: the 2007 ISCD Official Positions. J Clin Densitom 2008;11:123e62.

Cite this article as: Yuan Y, Zhang P, Tian W, Deng X, Yue R, Ge X, Li X. Application of bone turnover markers and DXA and QCT in an elderly Chinese male population. Ann Palliat Med 2021;10(6):6351-6358. doi: 10.21037/apm-21-612
16. American College of Radiology. ACR practice guideline for the performance of quantitative computed tomography (QCT) bone Densitometry (resolution 33). Reston, VA, USA, 2008.

17. Geusens $P$, Dinant $G$. Integrating a gender dimension into osteoporosis and fracture risk research. Gend Med 2007;4 Suppl B:S147-61.

18. Adler RA. Osteoporosis in men: insights for the clinician. Ther Adv Musculoskelet Dis 2011;3:191-200.

19. Liu MY, Li CL, Pei Y, et al. Diagnostic values of selfassessment tool for Asians for osteoporosis in aged men. Zhonghua Yi Xue Za Zhi 2011;91:2112-5.

20. Wu Q, Tao GS, Liu XL, et al. The determination of dual energy X-ray bone mineral density and the investigation of osteoporosis morbidity on people in Beijing District. Chin J Osteoporosis 1995;1:76-80.

21. Mazzaglia PJ, Berber E, Kovach A, et al. The changing presentation of hyperparathyroidism over 3 decades. Arch Surg 2008;143:260-6.

22. Braverman ER, Chen TJ, Chen AL, et al. Age-related increases in parathyroid hormone may be antecedent to both osteoporosis and dementia. BMC Endocr Disord 2009;9:21.

23. Gomella LG, Haist SA. "Chapter 4. Laboratory Diagnosis: Chemistry, Immunology, Serology” (Chapter). Clinician's Pocket Reference: The Scut Monkey, 11e. Available online: http://www.accessmedicine.com/content. aspx?aID=2699454

24. Williams C, Sapra A. Osteoporosis Markers. 2021 May 10. In: StatPearls [Internet]. Treasure Island (FL): StatPearls Publishing; 2021. PMID: 32644732. 